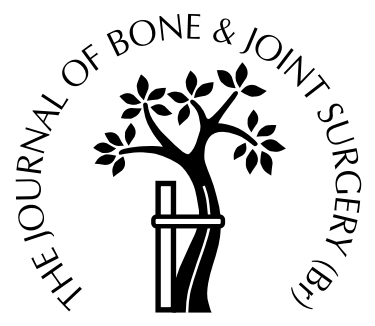

\title{
Sensory dysfunction in the great toe in hallux valgus
}

\author{
M. L. Herron, S. Kar, D. Beard, P. Binfield \\ From the Warwick District General Hospital and the Nuffield Orthopaedic Centre, \\ Oxford, England
}

I njury to the dorsomedial cutaneous nerve in the foot may occur after operations for hallux valgus. Pressure neuropathy before operation is also described but remains largely unexplored. We have investigated the incidence of sensory deficit in the great toe before operating for hallux valgus and examined to what extent any deficit was related to the degree of angulation of the joint.

Forty-three patients with a total of 61 great toes with hallux valgus presenting for consideration of surgical correction had their sensation tested in pre-designated zones using a five-filament set of Semmes-Weinstein monofilaments. These allowed good inter-observer reliability with an intra-class correlation coefficient of 0.84 .

Sensory symptoms were noted by only $21 \%$ of the patients, a measurable reduction in sensation by one monofilament grade or more was found in an additional $44 \%$. No relationship was found between the degree of sensory loss and the degree of angulation.

Patients with symptomatic hallux valgus may have sensory loss in the toe without being aware of it. Normal subjective sensation does not reliably predict normal sensory function. Given the potentially high rates of nerve damage following operations for hallux valgus, we recommend objective sensory testing as part of routine assessment before surgery.

J Bone Joint Surg [Br] 2004;86-B:54-7.

Received 30 April 2002; Accepted after revision 8 July 2003

M. L. Herron, FRCS Orth, Specialist Registrar in Orthopaedics

S. Kar, MRCS, Senior House Officer in Orthopaedics

P. Binfield, FRCS Orth, Consultant Orthopaedic Surgeon

Department of Orthopaedic and Trauma Surgery, Warwick District General Hospital, Lakin Road, Warwick CV34 5BW, UK.

D. Beard, DPhil, Senior Fellow

Nuffield Department of Orthopaedic Surgery, Nuffield Orthopaedic Centre, Windmill Road, Headington, Oxford OX3 7LD, UK.

Correspondence should be sent to Mr M. L. Herron at The Royal Orthopaedic Hospital, Woodlands, Northfield, Birmingham B31 2AP, UK.

(C)2004 British Editorial Society of Bone and Joint Surgery doi:10.1302/0301-620X.86B1.13502 \$2.00
Pressure neuropathy may sometimes contribute to the symptoms in hallux valgus, ${ }^{1,2}$ but little information is available as to its incidence or the identity of the nerves affected. One case report ${ }^{3}$ describes the symptoms in hallux valgus as specifically 'neuritic' with a successful result following neurolysis of an entrapped dorsomedial cutaneous nerve.

Recent interest has focused on the exact course and variations in the anatomy of the dorsomedial cutaneous nerve of the hallux, a terminal branch of the superficial peroneal nerve $e^{4,5}$ because of a reported incidence of iatrogenic injury of up to $45 \%$. $^{6,7}$ Having encountered a number of patients presenting with medially located parasthesiae in the big toe as a symptom of hallux valgus, we undertook a study of the cutaneous sensation prior to surgery. The null hypothesis proposed was that there was no significant difference between sensation proximal and distal to the angular deformity at the metatarsophalangeal (MTP) joint in hallux valgus. In addition, on the basis that any sensory disturbance might be secondary to nerve traction around the bunion deformity, we looked for any quantitative relationship between the extent of the deformity and sensory loss. We also investigated whether those who complained of sensory disturbance had a greater degree of dysfunction than patients with a demonstrable deficit of which they were unaware.

\section{Patients and Methods}

Forty-three consecutive patients presenting with hallux valgus were recruited to the study. Those with altered peripheral sensation, due either to the potential for peripheral neuropathy, a history suggestive of more proximal nerve entrapment or previous surgery to the hallux were excluded. All the patients were female with a mean age of 56 years (35 to 73 ); 18 had bilateral hallux valgus and 25 unilateral, giving a total of 61 feet. Of these, 33 were right and 28 were left. In addition, 12 contralateral normal feet were also studied in the same patients.

All patients were asked whether they were aware of sensory symptoms and if these were regarded as a contributory factor in their disability. Sensory testing was carried out using a Semmes-Weinstein monofilament kit, the WEST (Connecticut Bio-instruments Inc, Danbury, Connecticut/ Smith and Nephew Healthcare, Cambridge, UK) (Fig. 1). 


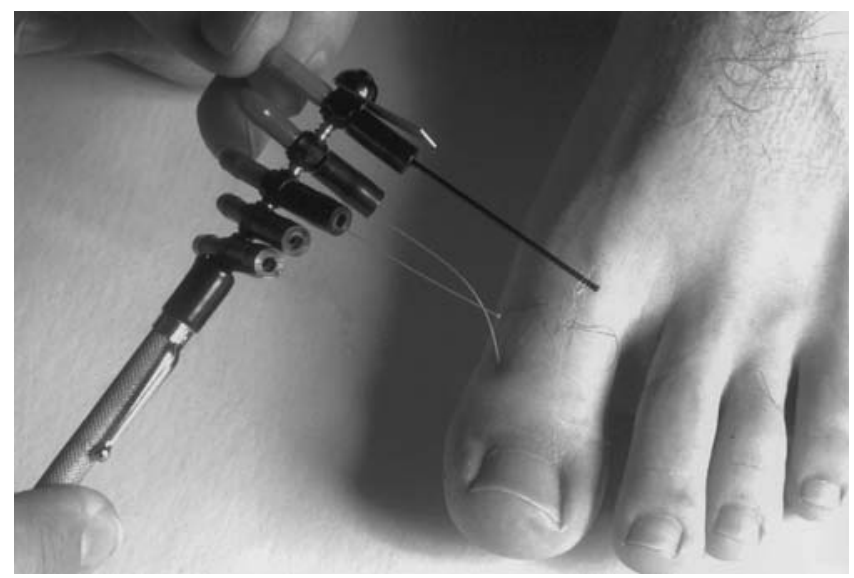

Fig. 1

The Semmes-Weinstein five monofilament testing kit.

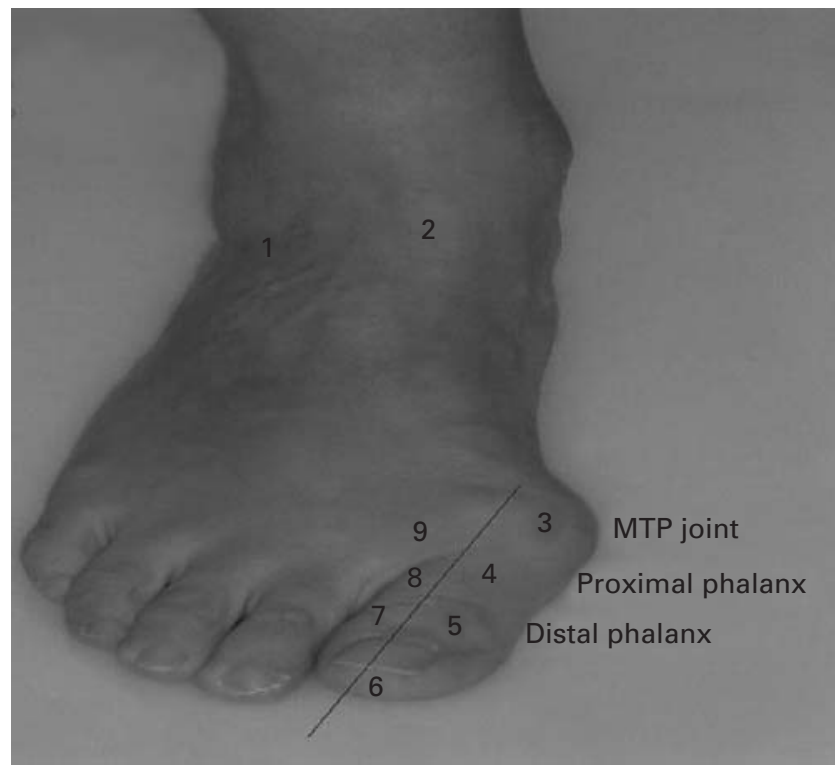

Fig. 2

The distribution of the sites tested in the foot (1, sural nerve; 2 to 4 , superficial peroneal nerve; 5 to 7 , medial plantar nerve; 8 to 9 , deep peroneal nerve).

This comprises a single pen-sized handle with five rotatable monofilaments of regulated length and diameter. A repeatable force stimulus is delivered by applying each monofilament perpendicular to the test site slowly until it bends and leaving it in situ for one second. This tests light touch sensation. Monofilaments were not calibrated between cases on a top loading balance since we were looking at patterns of sensory alteration within individual feet and did not compare absolute values between feet.

Testing was performed consistently in all feet by MLH and SK and as recommended by previous studies. ${ }^{8,9}$ The smallest filament was used first with progression to the largest. Patients were seated for testing with the foot elevated on a low stool and were blinded to the testing. A positive threshold was recorded when a patient could feel the stimulus and locate its position. The same sites were recorded in each foot (Fig. 2) and corresponded to recognised territory for the superficial and deep peroneal, medial plantar and sural nerves. In the area of the bunion (zone 3 ) the thickened skin over the deformity was avoided if possible.

A control zone (2), supplied by the superficial peroneal nerve, was established proximal to the deformity in order to allow comparison with sensory changes at and distal to it. A secondary control zone (1), supplied by the sural nerve was also used as a check. It was assumed that these two dorsal areas, both equidistant from the ankle, would have similar sensory function and so detect any significant proximal disturbance of the superficial peroneal nerve in the lower leg. Absence of a significant difference in sensory levels between the two dorsal control zones enabled valid comparison between the test zones ( 3 to 9 ) and the appropriate control zone (2).

Zones 3, 4 and 5 were located to the medial side of the dorsal midline of the hallux and comprised normal dorsal skin. Zone 3 was located at the MTP joint, zone 4 at the proximal phalanx and zone 5 at the distal phalanx. Zones 9, 8 and 7 were located directly opposite zones 3,4 and 5 on the lateral side of the midline and comprised dorsal skin. Zone 6 was an area of planter skin on the tip of the distal phalanx.

A separate group of ten control feet from normal subjects, and not included in the analysis, were also examined to allow quantification of the inter-observer variability with the monofilament testing kit. Standing radiographs were obtained to allow measurement of the hallux valgus and inter-metatarsal angles, as part of the routine assessment of the forefoot. These films also allowed us to assess the relationship between the degree of the radiographic deformity and any reduction in sensation. Given the reduction in light touch, which occurs with ageing, we also looked for evidence of any relationship between age and sensory appreciation at each zone.

\section{Analysis}

Each foot was analysed independently to determine whether there was any alteration in sensitivity at or distal to the hallux valgus deformity. A non-parametric Wilcoxon matched pairs test was performed in both the normal and affected feet to test for significance between the two control zones (1 and 2).

Preliminary analysis established that the sensory function of the superficial peroneal nerve proximal to the hallux deformity (zone 2) was unaffected and identical to that of the sural nerve at the same level. This allowed the control zone 2 to be used as the control throughout. A non-parametric Wilcoxon matched pairs test was performed to test for significance between zone 2 , and each of the other sites 3 to 9 distal to it. Each of the sites was also tested separately 
against each other to check for possible unexplained significant differences between zones. A Friedman non-parametric test was used to identify any differences between any zone over time. The hypothesis that patients who reported symptoms of sensory dysfunction in the hallux had a more significant sensory deficit than those who did not was tested using a non-parametric Mann-Whitney U test.

The hypothesis that changes in sensation were accounted for by age was tested by looking at the relationship between age and sensory grade at each zone using Spearman's correlation coefficient. The relationship between the degree of deformity, as measured by both the hallux valgus and the intermetatarsal angles and the severity of any sensory deficit was investigated using the coefficient of correlation. Interobserver error was quantified for each zone using intraclass correlation coefficients.

\section{Results}

There was no significant difference between control zone 1 and 2 in either feet with hallux valgus $(p=0.7)$ or in those without $(\mathrm{p}=0.65)$.

In the feet with hallux valgus there were significant differences in sensitivity between the distribution of the dorsomedial cutaneous nerve proximal to the MTP joint in zone 2 (mean 2.82, SD 0.7) and between zones 3 (mean 3.7, SD 0.9, $\mathrm{p}=0.0001)$ and 4 (mean 3.12, SD 0.67, p = 0.017). There was a decrease in sensation at and distal to the angular deformity of the MTP joint, limited to the proximal phalanx. This pattern was not present in the normal feet, which showed no significant difference between any of the zones studied. There were no other significant differences between any other zones.

The mean angular deformity of the hallux group was an intermetatarsal (IM) angle of $14^{\circ}$ and an hallux valgus (HV) angle of $35^{\circ}$. There was no correlation between the degree of angulation and the sensory deficit. The mean angular deformity of the contralateral normal feet was an intermetatarsal angle of $10^{\circ}$ and a hallux valgus angle of $19^{\circ}$. The difference between the two groups was significant $(p=0.001$ for both parameters, with a $95 \%$ confidence interval of the difference 1.9 to 5.9 for the IM angle and 8.6 to 22 for the HV angle).

When objective sensation in patients with hallux valgus who had described sensory symptoms (13 patients and 17 feet) was compared with those who did not (30 patients and 44 feet), no significant differences were found. Of patients who mentioned sensory symptoms, five (38\%) regarded these as part of the reason for seeking referral.

There was no evidence of a relationship between age and sensory change.

\section{Discussion}

When the level of sensation on the dorsal aspect of the hallux was measured in a group of patients with hallux valgus, there was clear evidence of a significant reduction in the region of the deformity. This decrease was only present in the territory of the dorsomedial cutaneous nerve at the bunion and the adjacent proximal phalanx, and was more marked over the bunion (Fig. 2). This differential effect may not be wholly accounted for by a differential reduction in nerve function, but may also reflect the effect of the generally calloused skin of the bunion area on perceived sensation. The medial side of the distal phalanx is supplied by the medial plantar nerve and is therefore not affected. ${ }^{10}$ Traction around the bunion deformity, direct pressure between the bunion and the shoe and recurrent inflammation of the bursal tissue may affect the dorsomedial cutaneous nerve but not the medial plantar. Although general figures are available for dorsal sensation,,${ }^{9,11}$ no detailed study allowing comparison exists.

Chronic traction of a nerve around a bony prominence resulting in dysfunction is well recognised particularly in the case of the ulnar nerve at the medial epicondyle and the common peroneal nerve at the neck of the fibula. This study has shown that dysfunction of the dorsomedial cutaneous nerve can be demonstrated in hallux valgus, but we have not been able to demonstrate a relationship between the degree of angular deformity and severity of sensory loss.

The location of the dorsomedial cutaneous nerve in relation to incisions on the hallux $x^{4,5}$ has resulted in a high incidence of post-operative injury ${ }^{6}$ with the recognition that the resulting neuromas may lead to poor outcomes. ${ }^{10,12} \mathrm{We}$ have shown that in $65 \%$ of great toes some deficit in sensation is present before operation. Unless this has been assessed before surgery, dysfunction after operation cannot be absolutely ascribed to the operation. Although $21 \%$ of the patients complained of symptoms related to nerve dysfunction, this was demonstrated in another $44 \%$. Previous authors ${ }^{6}$ have also noted this disparity between lower subjective and higher objective incidences of sensory dysfunction of the hallux after operation.

Testing for two-point discrimination is often used in clinical assessment of sensory dysfunction but has limitations given the difficulty in applying similar pressures between tests and observers. ${ }^{8}$ Its use in compressive neuropathies, where the cortical representation which the technique tests is expected to be normal, has been questioned. ${ }^{13}$ The Semmes-Weinstein monofilaments provide a controlled, objective and reproducible force stimulus. ${ }^{8}$ Their use as a screening tool for critical sensory impairment in diabetic feet is established. ${ }^{14}$ More recently, they have been used to document general sensory thresholds of normal foot sensation. ${ }^{9}$ The method is easily applied and reproducible. Given the potentially high incidence of intraoperative damage, we recommend its consideration as a routine part of the pre-operative assessment for hallux valgus.

No benefits in any form have been received or will be received from a commercial party related directly or indirectly to the subject of this article. 


\section{References}

1. Jahss MJ. Disorders of the foot. Philadelphia: WB Saunders, 1982; 1173.

2. Root ML. Normal and abnormal function of the foot: Clinical Biomechanics. Vol 12:419. Los Angeles: Clinical Bio-mechanics Corp, 1977.

3. Rosen JS, Grady JF. Neuritic bunion syndrome. J Am Podiatr Med Assoc 1986;76:641-4.

4. Miller RA, Hartman G. Origin and course of the dorsomedial cutaneous nerve to the great toe. Foot Ankle Int 1996;17:620-2.

5. Solan MC, Lemon M, Bendall SP. The surgical anatomy of the dorsomedial cutaneous nerve of the hallux. J Bone Joint Surg [Br] 2001; 83-B:250-2.

6. Campbell DA. Sensory nerve damage during surgery on the hallux. J R Coll Surg Edinb 1992;37:422-4.

7. Meier PJ, Kenzora JE. The risks and benefits of distal first metatarsal osteotomies. Foot Ankle 1985;6:7-17.
8. Bell-Krotoski JA, Burford WL. The force/time relationship of clinically used sensory testing instruments. J Hand Therapy 1997;297-309.

9. Jeng C, Michelson J, Mizel M. Sensory thresholds of normal human feet. Foot Ankle Int 2000;21:501-4.

10. Kenzora JE. Sensory nerve neuromas - leading to failed foot surgery. Foot Ankle 1986;7:110-7.

11. Bell-Krotoski J. Advances in sensibility evaluation. Hand Clin 1991;7:527-46.

12. Kkenzora JE. Symptomatic incisional neuromas on the dorsum of the foot. Foot Ankle Int 1984;5:2-15.

13. Scrossan J. Nerves. In: Barton N, Mulligan $\mathrm{P}$, eds. The upper limb and hand. Philadelphia: WB Saunders, 1999:211.

14. Kumar S, Fernando DJ, Veves A, et al. Semmes-Weinstein monofilaments: a simple effective and inexpensive screening device for identifying diabetic patients at risk for foot ulceration. Diabetes Res Clin Pract 1991;13:63-8. 